\title{
Sequence conservation analysis and in silico human leukocyte antigen-peptide binding predictions for the Mtb72F and M72 tuberculosis candidate vaccine antigens
}

\author{
Marie-Cécile Mortier ${ }^{*}$, Erik Jongert, Pascal Mettens and Jean-Louis Ruelle
}

\begin{abstract}
Background: Requisites for an efficacious tuberculosis (TB) vaccine are a minimal genomic diversity among infectious Mycobacterium tuberculosis strains for the selected antigen, and the capability to induce robust T-cell responses in the majority of human populations. A tool in the identification of putative T-cell epitopes is in silico prediction of major histocompatibility complex (MHC)-peptide binding. Candidate TB vaccine antigen Mtb72F and its successor M72 are recombinant fusion proteins derived from Mtb32A and Mtb39A (encoded by Rv0125 and Rv1196, respectively). Adjuvanted Mtb72F and M72 candidate vaccines were shown to induce CD4 ${ }^{+}$T-cell responses in European, US, African and Asian populations.
\end{abstract}

Methods: Sequence conservation of Mtb32A, Mtb39A, Mtb72F and M72 among 46 strains (prevalent Mycobacterium strains causing human TB disease, and H37Ra) was assessed by multiple alignments using ClustalX. For Mtb32A, Mtb39A and Mtb72F, 15-mer human leukocyte antigen (HLA)-class II-binding peptides were predicted for 158 DRB1 alleles prevailing in populations with high TB burden, 6 DRB3/4/5, 8 DQ and 6 DP alleles, using NetMHCIIpan-3.0. Results for 3 DRB1 alleles were compared with previously published allele-matched in vitro binding data. Additional analyses were done for M72. Nonameric MHC class I-binding peptides in Mtb72F were predicted for three alleles representative of class I supertypes A02, A03 and B07, using seven prediction algorithms.

Results: Sequence identity among strains was $\geq 98 \%$ for each protein. Residue changes in Mtb39A comprised primarily single residue or nucleotide insertions and/or deletions in repeat regions, and were observed in $67 \%$ of strains. For Mtb72F, 156 DRB1, 6 DRB3/4/5, 7 DQ and 5 DP alleles were predicted to contain at least one MHC class II-binding peptide, and class I-binding peptides were predicted for each HLA-A/B allele. Comparison of predicted MHC-II-binding peptides with experimental data indicated that the algorithm's sensitivity and specificity were variable among alleles.

Conclusions: The sequences from which Mtb72F and M72 are derived are highly conserved among representative Mycobacterium strains. Predicted putative T-cell epitopes in M72 and/or Mtb72F covered a wide array of HLA alleles. In silico binding predictions for class I- and II-binding putative epitopes can be complemented with biochemical verification of HLA binding capacity, processing and immunogenicity of the predicted peptides.

\section{Background}

Genetic variation in antigen components of vaccines at the pathogen level as well as in the host (the latter relating to the polymorphism in the human leukocyte antigen [HLA]), are major determinants for human vaccine efficacy. Mycobacterium tuberculosis (Mtb) is the causative

\footnotetext{
* Correspondence: marie-cecile.mortier@gsk.com

GSK Vaccines, Rue de l'Institut 89, 1330 Rixensart, Belgium
}

agent of tuberculosis (TB) disease. Requisites for an efficacious TB vaccine to be used in populations world-wide are a minimal genomic variation of the target antigen among different Mtb strains, and the ability of the antigen's contents of peptides to bind to the largest possible subset of HLA alleles in the host populations. The TB vaccine should also be able to induce robust T-cell responses [1]. While $\mathrm{CD} 4^{+} \mathrm{T}$ cells are thought to take center stage in the protection against $\mathrm{Mtb}$ infection, $\mathrm{CD} 8^{+} \mathrm{T}$ cells are also

\section{Biomed Central}

(c) 2015 Mortier et al. Open Access This article is distributed under the terms of the Creative Commons Attribution 4.0 International License (http://creativecommons.org/licenses/by/4.0/), which permits unrestricted use, distribution, and reproduction in any medium, provided you give appropriate credit to the original author(s) and the source, provide a link to the Creative Commons license, and indicate if changes were made. The Creative Commons Public Domain Dedication waiver (http://creativecommons.org/publicdomain/zero/1.0/) applies to the data made available in this article, unless otherwise stated. 
assumed to contribute to immunity against TB. Consequently, the selection of a vaccine antigen is in part focused on the abundance of HLA class I and class II-binding epitopes, which may be indicative of its immunogenicity.

HLA-peptide binding is a prerequisite for a putative epitope, linked to its correct processing and immunogenicity, and in silico prediction of this binding has become a tool in vaccine design. Predictive computer algorithms are generally trained by databases of experimentally validated epitopes with T-cell stimulation potential, such as SYFPEITHI [2] or the Immune Epitope Database (IEDB) [3]. For Mtb, the IEDB lists predominantly major histocompatibility complex class II (MHC-II)-restricted epitopes of the H37Rv strain [4], and the number of identified MHC class I (MHC-I)-restricted Mtb epitopes is limited [5]. Moreover, few Mtb epitopes have been identified for alleles prevailing in populations in TB-endemic regions. The known epitopes that are targeted by human T cellmediated immune responses cover only $4 \%$ of the Mtb proteome [6].

The pe/ppe gene family, which includes $\sim 170$ members, comprises approximately $10 \%$ of the Mtb genome [7]. It has been suggested that proteins secreted by these genes aid in Mtb infection [8], and that Mtb39A (encoded by Rv1196/ppe18) has a role in Mtb virulence $[9,10]$. Since infection can induce $\mathrm{CD}^{+}$and $\mathrm{CD}^{+} \mathrm{T}$-cell responses to various PE/PPE proteins, some of these proteins have been evaluated as potential TB vaccine antigens $[7,11]$. The vaccine antigen $\mathrm{Mtb} 72 \mathrm{~F}$ is a recombinant polyprotein derived from the H37Rv-expressed proteins Mtb39A and Mtb32A (the latter protein is a putative serine protease encoded by $p e p A / R v 0125)$ [12]. Both genes are present in both virulent and avirulent strains of the Mtb complex, and in bacille Calmette-Guérin (BCG) [13, 14]. The Mtb72F construct was generated by linking the carboxyl-terminal fragment of Mtb32A (Mtb32 $)$, Mtb39A, and the amino-terminal fragment of Mtb32A excluding the signal sequence (Mtb32 $)$ [12]. In a clinical study, the vaccine candidate Mtb72F induced comparable magnitudes of Mtb32A- and Mtb39Aspecific $\mathrm{CD} 4^{+} \mathrm{T}$-cell responses [15]. Collectively, at least 10 $\mathrm{CD}^{+}{ }^{+}$or $\mathrm{CD}^{+} \mathrm{T}$-cell epitopes (which were recognized by PBMC from human PPD-positive donors) have been identified in Mtb39A [14, 16], and a mouse $\mathrm{CD}^{+}$T-cell epitope was identified within ${\mathrm{Mtb} 32_{\mathrm{C}}}_{[}[12,17]$.

Several Mtb72F peptides have been experimentally characterized to bind to one of three common HLA class II alleles (DRB1*01:01, 15:01 and 04:01) [18], or to the HLA class I allele B44 [16]. The successor of Mtb72F, M72, was generated from the Mtb72F sequence by introducing a point mutation in order to improve the long-term stability of the purified bulk of Mtb72F, and by modification of the $\mathrm{N}$-terminal poly-histidine (poly-His) sequence. In clinical studies, these modifications were shown to have no effect on the antigen's immunogenicity with respect to cell- mediated or humoral responses [19]. Indeed, antigenspecific $\mathrm{CD}^{+}{ }^{+} \mathrm{T}$-cell and antibody responses were elicited by AS02-adjuvanted Mtb72F vaccines in various adult populations [15, 19-21], and by AS01-adjuvanted M72 vaccines in populations of healthy adults in TB-endemic and non-endemic regions [19, 22, 23], HIV-1-infected adults in Switzerland [24], and healthy infants in The Gambia [25]. Consequently, M72 has been selected for further vaccine development.

The objectives of this in silico study were (1), to assess the sequence conservation of Mtb32A, Mtb39A, Mtb72F and M72, and (2), to predict MHC-I binding for nonameric peptides in Mtb72F, and MHC-II binding for 15-mer peptides in Mtb72F, Mtb32A and Mtb39A. The predictions focused on Mtb72F rather than M72 in order to allow comparison with previous prediction studies [26, 27], and additional evaluations were performed to assess the impact of the alterations introduced in Mtb72F to generate M72. MHC-II-binding predictions were evaluated for DRB1 alleles prevailing in populations with a high TB burden, and for DRB3/4/5, DP and DQ alleles. Last, we compared our prediction results obtained for three DRB1 alleles with allele-matched, experimentally derived MHC-II binding data described in [18].

Our findings suggested that the Mtb72F and M72 sequences are highly conserved among representative $\mathrm{Myco-}$ bacterium strains and that the putative T-cell epitopes predicted for these two proteins cover a wide array of HLA class I and II alleles, while highlighting the limitations of in silico epitope predictions in general.

\section{Results}

Sequence conservation analysis for Mtb32A, Mtb39A, Mtb72F and M72

Using sequence similarity search programs, we assessed the similarity of H37Rv-derived Mtb32A and Mtb39A among genomes of Mycobacterium strains causing human TB disease, and H37Ra. For the 40 strains for which both sequences were available, similarity was also evaluated for the Mtb72F and M72 sequences constructed in silico. Furthermore, the presence of nucleotide and amino acid changes was evaluated for Mtb39A, given the relatively high frequency of sequence polymorphism reported for this protein $[28,29]$. The strains evaluated included clinical isolates and representatives of all presently available Mtb strains, and comprised representatives of five of the six geographical lineages identified in a molecular phylogeny of the Mtb complex, which cover the predominant part of the Mtb geographical distribution [30].

For Mtb39A, we observed mainly single residue or nucleotide insertions and/or deletions with minor consequences for amino acid sequences, although amino acid changes were detected in $67 \%$ of strains assessed (Table 1). Indeed, amino acid sequence comparisons revealed an 
Table 1 Similarity of Mtb32A, Mtb39A, Mtb72F and M72 among selected Mycobacterium strains

\begin{tabular}{|c|c|c|c|c|c|c|c|c|c|c|}
\hline \multirow[t]{3}{*}{ Lineage $^{a}$} & \multirow[t]{3}{*}{ Strains } & \multirow{3}{*}{$\begin{array}{l}\text { NCBI Accession } \\
\text { number }\end{array}$} & \multicolumn{4}{|l|}{ Mtb39A } & \multirow{3}{*}{$\begin{array}{l}\text { Mtb39A } \\
\% \text { identit }\end{array}$} & \multirow{3}{*}{$\begin{array}{l}\text { Mtb32A } \\
y\end{array}$} & \multirow[t]{3}{*}{ Mtb72F } & \multirow[t]{3}{*}{ M72 } \\
\hline & & & \multirow{2}{*}{$\begin{array}{l}\text { No. of } \\
\text { aa changes }\end{array}$} & \multicolumn{3}{|c|}{ No. of aa/nt, position (pos) } & & & & \\
\hline & & & & Insertions & Deletions & Frameshifts & & & & \\
\hline \multirow[t]{4}{*}{1} & $\mathrm{~T} 17$ & PRJNA55273 & 3 & & & & 99.23 & 94.37 & 96.79 & 96.65 \\
\hline & T46 & PRJNA55875 & 3 & & & & $99.23^{b}$ & - & - & - \\
\hline & T92 & PRJNA55099 & 3 & & & & 99.23 & 100 & 99.58 & 99.44 \\
\hline & EAS054 & PRJNA55133 & 117 & $1 \mathrm{nt}$, pos 811 & & 1, pos 270-391 & 68.92 & 100 & 84.37 & 84.23 \\
\hline \multirow[t]{5}{*}{2} & 94_M4241A & PRJNA55095 & 0 & & & & 100 & 100 & 100 & 99.86 \\
\hline & $\begin{array}{l}210 \\
\text { (Mtb Beijing) }\end{array}$ & PRJNA42617 & 30 & & 1 aa, pos 274 & & 91.3 & 100 & 95.4 & 95.26 \\
\hline & 02_1987 & PRJNA55097 & 1 & & & & 99.74 & 100 & 99.86 & 99.72 \\
\hline & T85 & PRJNA55131 & 1 & & & & 99.74 & 100 & 99.86 & 99.72 \\
\hline & $\begin{array}{l}\text { W-148 } \\
\text { (Mtb. Beijing;MDR) }\end{array}$ & PRJNA182020 & 1 & & & & 99.74 & 100 & 99.86 & 99.72 \\
\hline 3 & - & & & & & & - & - & - & - \\
\hline \multirow[t]{8}{*}{4} & CDC1551 & PRJNA57775 & 0 & & & & 100 & 100 & 100 & 99.86 \\
\hline & C & PRJNA54359 & 0 & & & & $100^{b}$ & - & - & - \\
\hline & F11 & PRJNA58417 & 1 & & & & 99.74 & 100 & 99.86 & 99.72 \\
\hline & GM1503 & PRJNA55271 & - & - & - & - & - & $100^{b}$ & - & - \\
\hline & $\mathrm{H} 37 \mathrm{Ra}^{\mathrm{C}}$ & PRJNA58853 & 62 & & 1 nt, pos 922 & 1, pos 309-391 & 82.28 & 100 & 90.56 & 90.42 \\
\hline & Haarlem & PRJNA54453 & 0 & & & & 100 & 100 & 100 & 99.86 \\
\hline & KZN1435 (MDR) & PRJNA59069 & 0 & & & & 100 & 100 & 100 & 99.86 \\
\hline & 98R604INHRIFEM & PRJNA55399 & 0 & & & & 100 & 100 & 100 & 99.86 \\
\hline 5 & $\begin{array}{l}\text { CPHL_A } \\
\text { (M. africanum) }\end{array}$ & PRJNA55877 & 4 & & 1 aa, pos 274 & & 98.72 & 100 & 99.3 & 99.16 \\
\hline 6 & $\begin{array}{l}\text { K85 } \\
\text { (M. africanum) }\end{array}$ & PRJNA55879 & 3 & 2 aa, pos 162 & 1 aa, pos 274 & & 98.48 & 100 & 99.17 & 99.03 \\
\hline \multirow[t]{20}{*}{ n.d. } & SUMu001 & PRJNA51927 & 8 & & 1 aa, pos 274 & & 97.70 & 100 & 98.75 & 98.61 \\
\hline & SUMu002 & PRJNA51925 & - & - & - & - & - & $100^{b}$ & - & - \\
\hline & SUMu003 & PRJNA51931 & 2 & & & & 99.49 & 100 & 99.72 & 99.58 \\
\hline & SUMu004 & PRJNA51933 & 2 & & 1 aa, pos 274 & & 99.23 & 100 & 99.58 & 99.44 \\
\hline & SUMu005 & PRJNA51935 & 2 & & 1 aa, pos 274 & & 99.23 & 100 & 99.58 & 99.44 \\
\hline & SUMu006 & PRJNA91937 & 2 & & & & 99.49 & 100 & 99.72 & 99.58 \\
\hline & SUMu008 & PRJNA51941 & - & - & - & - & - & $100^{b}$ & - & - \\
\hline & SUMu010 & PRJNA51945 & - & - & - & - & - & $100^{b}$ & - & - \\
\hline & KZN605 (XDR) & PRJNA54947 & 0 & & & & 100 & 100 & 100 & 99.86 \\
\hline & KZN4207 & PRJNA83619 & 0 & & & & 100 & 100 & 100 & 99.86 \\
\hline & KZNR506 (XDR) & PRJNA47489 & 0 & & & & 100 & 100 & 100 & 99.86 \\
\hline & $\begin{array}{l}\text { KZNV2475 } \\
\text { (MDR) }\end{array}$ & PRJNA47491 & 1 & & & & 99.74 & 100 & 99.86 & 99.72 \\
\hline & UT205 & PRJNA162183 & 0 & & & & 100 & 100 & 100 & 99.86 \\
\hline & BTB05-552 & PRJNA51871 & 0 & & & & 100 & 100 & 100 & 99.86 \\
\hline & BTB05-559 & PRJNA51873 & 0 & & & & 100 & 100 & 100 & 99.86 \\
\hline & $596-129$ & PRJNA51869 & 0 & & & & 100 & 100 & 100 & 99.86 \\
\hline & CCDC5079 & PRJNA161943 & 1 & & & & 99.74 & 100 & 99.86 & 99.72 \\
\hline & CCDC5180 & PRJNA161941 & 1 & & & & 99.74 & 100 & 99.86 & 99.72 \\
\hline & CTRI-2 & PRJNA161997 & 0 & & & & 100 & 100 & 100 & 99.86 \\
\hline & CTRI-4 (XDR) & PRJNA43175 & 3 & & & & 99.23 & 100 & 99.58 & 99.44 \\
\hline
\end{tabular}


Table 1 Similarity of Mtb32A, Mtb39A, Mtb72F and M72 among selected Mycobacterium strains (Continued)

\begin{tabular}{|c|c|c|c|c|c|c|c|c|c|c|}
\hline & R1207 (MDR) & PRJNA46669 & 1 & & & & 99.74 & 100 & 99.86 & 99.72 \\
\hline & X122 (pre-XDR) & PRJNA46667 & 1 & & & & 99.74 & 100 & 99.86 & 99.72 \\
\hline & NA-A0008 & PRJNA168604 & 6 & & & & 98.47 & 100 & 99.58 & 99.44 \\
\hline & NA-A0009 & PRJNA168605 & 3 & & & & 99.23 & 100 & 99.16 & 99.02 \\
\hline & HN878 & PRJNA46665 & 3 & & & & 99.23 & 100 & 99.58 & 99.44 \\
\hline & RGTB423 & PRJNA162179 & 2 & & & & 99.23 & 85.63 & 92.48 & 92.34 \\
\hline & RGTB327 & PRJNA157907 & 24 & 1 nt, pos 480 & 1 nt, pos 464 & 1, pos 139-155 & 93.91 & 100 & 96.67 & 96.53 \\
\hline & & & & 2 nt, pos 951 & 1 nt, pos 468 & 1, pos 314-316 & & & & \\
\hline & & & & $1 \mathrm{nt}$, pos 959 & 1 nt, pos 469 & & & & & \\
\hline & & & & & 1 nt, pos 470 & & & & & \\
\hline & & & & & $1 \mathrm{nt}$, pos 475 & & & & & \\
\hline $\begin{array}{l}\text { Affected Strains; } \\
\text { no }(\%)\end{array}$ & & & $28(67)$ & $3(7)$ & $8(19)$ & $3(7)$ & & & & \\
\hline $\begin{array}{l}\text { Average \% } \\
\text { identity }\end{array}$ & & & & & & & 98.08 & 99.55 & 98.71 & 98.57 \\
\hline
\end{tabular}

aLineages as defined in ref. [30]: no. 1: The Philippines / rim of the Indian Ocean; no. 2: East Asia; no. 3: India / East Africa; no. 4: Europe / Americas; no. 5 and no. 6: West African 1 and 2, respectively. n.d., not defined. MDR, multiple drug-resistant; (pre-)XDR, (pre-)extensively drug-resistant. aa, amino acid(s); nt, nucleotide(s). ${ }^{\mathrm{b}}$ Evaluations not performed for Mtb72F/M72 as a complete sequence was only available for one of the two genes. \% identity= (number of identical residues / length of alignment) x100, as calculated from multiple alignment comparisons. Mtb39A genome sequences were derived from complete genomes (chromosomes; highlighted in bold) or assembled partial genomes (scaffolds/contigs; normal font). 'Laboratory strain H37Ra was used as reference

average of $98 \%$ identity for Mtb39A, with $100 \%$ identity for 13 strains and at least $91 \%$ identity among all strains excluding EAS054 and H37Ra. The latter strains contained one frameshift each, both associated with major amino acid changes of 117 and 62 residues, respectively, and consequently also with lower similarity (69 and $82 \%$, respectively). Relative to Mtb39A, sequence identity was higher for Mtb32A (i.e., $99.6 \%$ on average, and $100 \%$ for 42 of the 44 strains assessed), consistent with earlier reports [28].

Mtb32A and Mtb39A sequences in 13 clinical isolates were identical to those in Mtb72F, and 84-99.9\% sequence identity was observed for the remaining strains. Among the three extensively drug-resistant (XDR), 1 pre$\mathrm{XDR}$ and four multiple drug-resistant (MDR) strains assessed, percentages identity for Mtb72F were at least $99.6 \%$. Overall, percentages identity were $0.14 \%(1 / 718)$ lower for M72 than for Mtb72F due to the introduced point mutation in M72, and exceeded $98 \%$ for both antigens in at least one strain per selected Mtb lineage.

MHC-II-binding peptides in Mtb32A, Mtb39A and Mtb72F A computerized algorithm (NetMHCIIpan-3.0) was used to compute MHC-II binding predictions for 15-mer peptides in Mtb72F and (H37Rv) Mtb32A and Mtb39A, for 158 HLA-DRB1, 6 DRB3/4/5, 8 DQ and 6 DP alleles. The DRB1 alleles were selected based on their presence in populations in four regions with a high TB prevalence (Northern, Southern and North-Eastern India and China) and in the native population of Sub-Saharan Africa, which has a high TB incidence. The 6 most common DRB3/4/5 alleles [31] and the subset of DQ/DP molecules that are present in at least $85 \%$ of populations worldwide [32, 33] were also included, and together our selection contained the full panel of 46 DR, DP and DQ alleles reported to collectively cover almost $90 \%$ of populations worldwide [31].

HLA-II binding peptides predicted for Mtb72F were found to cover a wide array of alleles (Additional file 1). For DRB1*15 and DRB1*16, which have been linked to susceptibility to TB in Indian populations [34, 35], we predicted an average of 64 binding peptides in Mtb72F per allele for the three defined Indian regions. Across geographical regions, the highest numbers of binding peptides for Mtb72F were predicted for DRB1*01:02, 01:04, 08:10, 08:06, 01:01 and 01:08, i.e., 533-468 peptides/allele, or $0.65-0.75$ /allele after normalization of the results with respect to the different protein lengths of Mtb32A, Mtb39A and Mtb72F (Fig. 1). Average numbers of predicted binding peptides per allele in Mtb72F were highest in Sub-Saharan Africa (163 per allele) followed by the individual Indian regions (115-143 per allele) and China (131 per allele) (Table 2). There was a trend for higher numbers of binding peptides for Mtb39A relative to Mtb32A.

Across regions, no peptides were predicted to bind to five alleles (DRB1*03:02, 04:07, 14:10, 14:14 and 14:44) for Mtb32A, and to two alleles (DRB1*16:04 and 03:02) for Mtb39A. Consequently, binding peptides were also not predicted for DRB1*16:04 and 03:02 for Mtb72F, since for DRB1*16:04 all binding peptides were predicted to be located in the Mtb32A signal sequence (which is absent in Mtb72F), while for DRB1*03:02 no binding peptides were predicted for any part of Mtb32A or Mtb39A. Reported allele frequencies in certain Sub-Saharan African, 


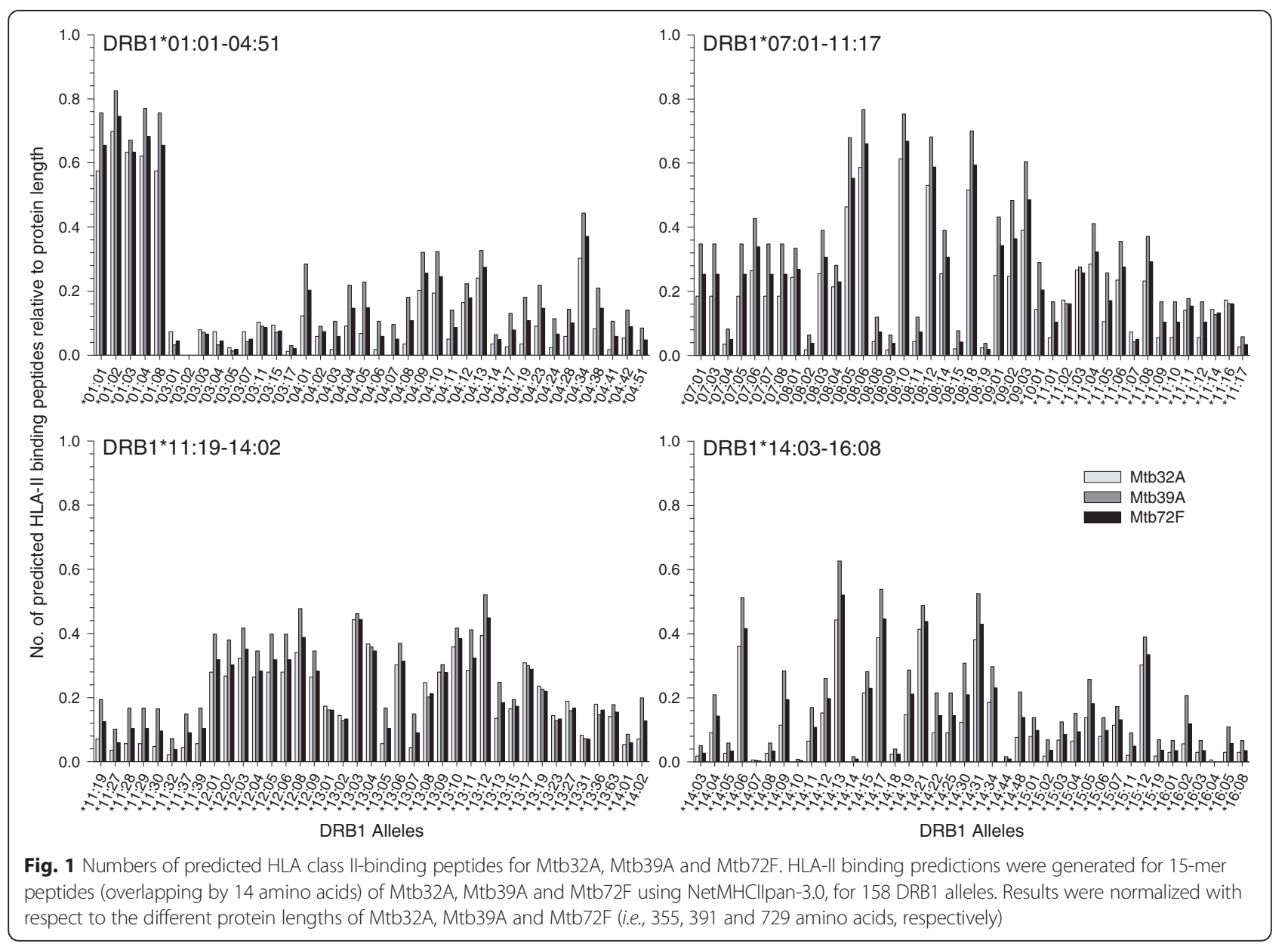

Chinese and Indian populations (as extracted from a public database [36]) are relatively low for DRB1*16:04 (ranging from 0.0-0.6 \%) and higher for DRB1*03:02 (ranging from 0.0-17.2 \%; Additional file 2).

For the 6 DRB3/4/5, 8 HLA-DQ and 6 HLA-DP molecules, we predicted up to 145,614 and 170 binding peptides in Mtb72F per allele, respectively (Table 3). Binding peptides in Mtb72F were predicted for each DRB3/4/5 and DP/DQ allele assessed, with the exception of DQA1"02:01-DQB1*02:01 (for which there were no prediction results for any of the four proteins assessed) and DPA1*02:02-DPB1*05:01 (for which all predicted peptides were located in the Mtb32A signal sequence). Worldwide, genotypic frequencies of DQA1*02:01-DQB1*02:01 and DPA1*02:02-DPB1*05:01 are $6 \%$ and $12 \%$, respectively [37], but for the populations assessed, no frequencies of these alleles have been reported in the used database (Additional file 2). For DQA1"02:01-DQB1*02:01 in haplotype with DRB1*07:01 (either alone or with DPB1*02:01, 03:01 or 17:01) frequencies in various populations ranged from 1-15\%. For DRB1*07:01, and thus also for the DRB1*07:01-
DQA1*02:01-DQB1*02:01 haplotypes, 181 peptides were predicted in Mtb72F (Additional file 1), with potentially higher numbers for haplotypes that also include DPB1*02:01 (Table 3).

\section{Impact of the alterations made to construct Mtb72F and} M72, on MHC-II binding predictions

Several alterations have been introduced into the native Mtb32A sequence in order to construct Mtb72F [12]; summarized in Table 4), and hence we have assessed the potential impact thereof on the prediction results for Mtb72F. We observed that the alterations resulted in a reduction of 42 predicted binding peptides, as well as in 1 and 18 additional predicted (non-Mtb) binding peptides owing to the addition of the poly-His tag and the hinge sequences respectively. As described in the previous section, no binding peptides were predicted after the alterations for one allele (DRB1*16:04) due to the deletion of the Mtb32A signal sequence. Additional evaluations were performed to assess the impact of the modifications introduced into Mtb72F in order to generate M72, 
Table 2 MHC class II-binding peptide predictions for common DRB1 alleles in high-TB burden regions

\begin{tabular}{|c|c|c|c|c|c|}
\hline \multirow{2}{*}{$\begin{array}{l}\text { Region/Alleles } \\
\text { assessed (N) }\end{array}$} & \multirow[t]{2}{*}{ Protein } & \multicolumn{2}{|c|}{ Predicted epitopes (N) } & \multicolumn{2}{|c|}{ DRB1 alleles without predicted epitope } \\
\hline & & Total & Average/allele & $\mathrm{N}$ & Allele \\
\hline Overall & Mtb32A & 8792 & 56 & 5 & ${ }^{*} 03: 02,{ }^{*} 04: 07,{ }^{*} 14: 10,{ }^{*} 14: 14,{ }^{*} 14: 44$ \\
\hline \multirow[t]{2}{*}{$(N=158)$} & Mtb39A & 14675 & 93 & 2 & ${ }^{*} 03: 02,{ }^{*} 16: 04$ \\
\hline & Mtb72F & 22065 & 140 & 2 & ${ }^{*} 03: 02,{ }^{*} 16: 04^{\mathrm{a}}$ \\
\hline China & Mtb32A & 5873 & 40 & 5 & ${ }^{*} 03: 02,{ }^{*} 04: 07,{ }^{*} 14: 10,{ }^{*} 14: 14,{ }^{*} 14: 44$ \\
\hline \multirow[t]{2}{*}{$(N=146)$} & Mtb39A & 12794 & 88 & 2 & ${ }^{*} 03: 02,{ }^{*} 16: 04$ \\
\hline & Mtb72F & 19109 & 131 & 2 & ${ }^{*} 03: 02,{ }^{*} 16: 04^{\mathrm{a}}$ \\
\hline N. India & Mtb32A & 2595 & 55 & 2 & ${ }^{*} 03: 02,{ }^{*} 04: 07$ \\
\hline \multirow[t]{2}{*}{$(N=47)$} & Mtb39A & 4516 & 96 & 1 & ${ }^{*} 03: 02$ \\
\hline & Mtb72F & 6733 & 143 & 1 & ${ }^{*} 03: 02^{\mathrm{a}}$ \\
\hline S. India & Mtb32A & 1087 & 43 & 0 & \\
\hline \multirow[t]{2}{*}{$(N=25)$} & Mtb39A & 1993 & 80 & 1 & ${ }^{*} 16: 04$ \\
\hline & Mtb72F & 2868 & 115 & 1 & ${ }^{*} 16: 04^{\mathrm{a}}$ \\
\hline N.E. India & Mtb32A & 2155 & 51 & 2 & ${ }^{*} 03: 02,{ }^{*} 04: 07$ \\
\hline \multirow[t]{2}{*}{$(N=42)$} & Mtb39A & 3517 & 84 & 1 & ${ }^{*} 03: 02$ \\
\hline & Mtb72F & 5298 & 126 & 1 & ${ }^{*} 03: 02^{\mathrm{a}}$ \\
\hline India (Total) & Mtb32A & 2807 & 37 & 2 & ${ }^{*} 03: 02,{ }^{*} 04: 07$ \\
\hline \multirow[t]{2}{*}{$(N=76)$} & Mtb39A & 4634 & 61 & 2 & ${ }^{*} 03: 02,{ }^{*} 16: 04$ \\
\hline & Mtb72F & 6876 & 90 & 2 & ${ }^{*} 03: 02,{ }^{*} 16: 04^{\mathrm{a}}$ \\
\hline S.S. Africa ${ }^{b}$ & Mtb32A & 4447 & 67 & 1 & ${ }^{*} 03: 02$ \\
\hline \multirow[t]{2}{*}{$(N=66)$} & Mtb39A & 6977 & 106 & 1 & ${ }^{*} 03: 02$ \\
\hline & Mtb72F & 10737 & 163 & 1 & ${ }^{*} 03: 02^{\mathrm{a}}$ \\
\hline
\end{tabular}

Prediction results represent the sum of the weak and strong binders. ${ }^{a}$ Allele frequencies in the populations assessed are included in Additional file $2 .{ }^{b}$ S.S. Africa, Sub-Saharan Africa, native population only

comprising the change of the $\mathrm{N}$-terminal poly-His sequence from $\mathrm{MHHHHHH}$ to $\mathrm{MHH}$, and the substitution of one serine residue for an alanine residue. Neither of the modifications resulted in a change in the number of alleles with at least one predicted putative epitope. No putative epitopes were predicted to be present in the MHH tag, and thus this modification of the poly-His tag resulted in the loss of the above described non-Mtb binding peptide predicted for the Mtb72F poly-His tag.

\section{Comparison of MHC-II binding predictions for 3 DRB1 alleles with experimental data}

We compared our prediction results for three common HLA class II alleles (DRB1*01:01, 04:01 and 15:01) with binding data obtained in vitro for Mtb32A and Mtb39A from Maeurer et al. [18]. In the latter study, the binding to soluble recombinant HLA-II monomers of three alleles was experimentally determined for 7466 15-mer Mtb peptides.

For Mtb72F predictions, the algorithm's sensitivity was higher for DRB1*01:01 than for DRB1*04:01 or
DRB1*15:01, as 74,28 and $33 \%$ respectively of the allele-matched experimentally identified binding peptides were also predicted by the algorithm, while the reverse was found for the specificities (which were 34, 81 and $90 \%$, respectively; Table 5). The same trends were observed for the prediction results obtained for Mtb32A and Mtb39A separately.

\section{MHC-I binding predictions for nonameric peptides in Mtb72F}

HLA I-binding 9-mer peptides in Mtb72F were predicted for the $A * 02: 01, A * 03: 01$ and $B * 07: 02$ alleles (representing the HLA-I supertypes A02, A03 and B07 respectively [38]). Collectively these supertypes have been reported to cover $80-90 \%$ of any given human population worldwide, regardless of ethnicity [39].

Using NetMHCpan-2.2, the combined numbers of strong and weak binders predicted for $A * 02: 01, A * 03: 01$ and $\mathrm{B}^{*} 07: 02$ were 23,2 and 15 , respectively (Additional file 3). The 11 strong binders predicted by this algorithm, and the 10 high-affinity binders predicted by NetCTLpan-1 were all restricted to $A^{*} 02: 01$ or $B^{*} 07: 02$. 
Table 3 Prediction of HLA II-binding peptides for DRB3/4/5, DP and DQ alleles

\begin{tabular}{|c|c|c|c|c|}
\hline HLA molecule & Mtb32A & Mtb39A & Mtb72F & M72 \\
\hline DRB3*01:01 & 18 & 7 & 31 & 31 \\
\hline DRB3*02:02 & 19 & 56 & 71 & 71 \\
\hline DRB3*03:01 & 51 & 82 & 125 & 125 \\
\hline DRB4*01:01 & 27 & 38 & 58 & 58 \\
\hline DRB5*01:01 & 32 & 85 & 109 & 109 \\
\hline DRB5*01:02 & 45 & 107 & 145 & 145 \\
\hline $\mathrm{DQA} 1{ }^{*} 05: 01-\mathrm{DQB} 1{ }^{*} 02: 01^{\mathrm{a}}$ & 14 & 35 & 51 & 51 \\
\hline $\mathrm{DQA} 1{ }^{*} 02: 01-\mathrm{DQB} 1{ }^{*} 02: 01^{\mathrm{b}}$ & 0 & 0 & 0 & 0 \\
\hline $\mathrm{DQA} 1^{*} 05: 01-\mathrm{DQB} 1^{*} 03: 01^{\mathrm{a}}$ & 291 & 325 & 614 & 610 \\
\hline DQA $1{ }^{*} 03: 01-D Q B 1 * 03: 02^{a}$ & 17 & 75 & 88 & 88 \\
\hline $\mathrm{DQA} 1{ }^{*} 04: 01-\mathrm{DQB} 1{ }^{*} 04: 02^{\mathrm{a}}$ & 15 & 75 & 87 & 88 \\
\hline $\mathrm{DQA} 1^{*} 01: 01-\mathrm{DQB} 1^{*} 05: 01^{\mathrm{a}}$ & 8 & 0 & 1 & 1 \\
\hline DQA1*01:02-DQB1*05:02 & 26 & 37 & 61 & 61 \\
\hline $\mathrm{DQA} 1{ }^{*} 01: 02-\mathrm{DQB} 1{ }^{*} 06: 02^{\mathrm{a}}$ & 161 & 262 & 392 & 392 \\
\hline $\mathrm{DPA} 1{ }^{*} 02: 01-\mathrm{DPB} 1{ }^{*} 01: 01^{\mathrm{c}}$ & 27 & 33 & 56 & 56 \\
\hline DPA $1^{*} 01: 03-D P B 1^{*} 02: 01^{c}$ & 20 & 23 & 39 & 39 \\
\hline DPA $1 * 01: 03-D P B 1 * 04: 01^{c}$ & 14 & 11 & 16 & 16 \\
\hline $\mathrm{DPA} 1{ }^{*} 01: 03-\mathrm{DPB} 1{ }^{*} 04: 02^{c}$ & 18 & 17 & 28 & 28 \\
\hline DPA $1^{*} 02: 02-D P B 1 * 05: 01^{b, c}$ & 8 & 0 & 0 & 0 \\
\hline DPA1*02:01-DPB1*14:01 & 50 & 128 & 170 & 170 \\
\hline
\end{tabular}

Prediction results represent the sum of the weak and strong binders. ${ }^{\text {a }}$ Allele is among the 6 HLA-DQ molecules that reportedly are present in $>85 \%$ of populations worldwide [32]. ${ }^{\mathrm{b}}$ For these alleles, no frequencies are reported in the populations assessed (Additional file 2). ${ }^{c}$ Allele is among the 5 HLA-DP molecules that reportedly are present in $>90 \%$ of populations worldwide [33]
Higher numbers of binding peptides (78 in total, of which 48 strong binders) were predicted by the combined 5 other algorithms that were used.

\section{Discussion}

This in silico study explored the sequence conservation of Mtb32A, Mtb39A, Mtb72F and the current candidate vaccine antigen M72, and predicted MHC-I and/or MHC-IIbinding peptides for these proteins. Our results led to two main conclusions. First, the Mtb32A and Mtb39A proteins (and thus also the Mtb72F and M72 constructs) appeared to be well conserved (with at least $98 \%$ identity) among the strains representative for the major part of the Mtb geographical distribution (including MDR, XDR and pre-XDR strains), with for Mtb39A a higher sequence conservation than previously reported in $[26,27]$. Second, the putative $\mathrm{CD}^{+} \mathrm{T}$-cell epitopes predicted for Mtb72F and M72 were shown to cover a broad range of HLA Class II DRB1, DRB3/4/5, DQ and DP alleles, and the putative $\mathrm{CD} 8^{+} \mathrm{T}$-cell epitopes predicted for Mtb72F covered each of the HLA-A or -B alleles assessed.

Hyperconservation has been reported for the large majority of human T-cell epitopes in the Mtb complex, although pe/ppe genes were excluded from that study [40]. Previously, $m t b 32 a$ sequences of H37Ra, H37Rv, Erdman and the clinical isolate CSU93 were found to be identical [13], and, extending these studies, Mtb32A was shown to be relatively well conserved among several clinical isolates, with amino acid changes in only $6 \%$ of the 225 investigated strains [28]. Consistently, our data revealed a high level of conservation for Mtb32A. For Mtb39A, our findings contrast with previous studies which suggested a substantial genetic variation for this protein, potentially associated with the homologous recombination with ppe19 and ppe60 $[28,29]$. Nonetheless, several of the minor insertions and/or deletions we observed in the Mtb39A

Table 4 Impact of the alterations introduced for the Mtb72F construction on the peptide binding predictions

\begin{tabular}{ll}
\hline Alteration introduced in Mtb72F & $\begin{array}{l}\text { Change in the numbers of } \\
\text { covered }^{\mathrm{a}} \text { alleles for Mtb72F }\end{array}$ \\
\hline Deletion of the Mtb32A signal sequence. & $\begin{array}{l}\text { Loss of } 1 \text { covered allele } \\
\text { containing } 28 \text { predicted } \\
\text { epitopes }\end{array}$
\end{tabular}

\section{Explanation}

Epitopes in the Mtb32A signal sequence were predicted for 149 of the 158 alleles assessed. For 148 of the 149 alleles, epitopes were also predicted for the other parts of the protein. Only for 1 allele (DRB1 ${ }^{*} 16: 04$ ) all 28 predicted epitopes were located in the Mtb32A signal sequence, and were thus not predicted for Mtb72F.
Splitting the Mtb32A sequence upstream and downstream of 'TAAS' sequence.

Addition of a poly-His tag $(\mathrm{MHHHHHH})$ at the Mtb32A C-terminal end.
No changes in the number of covered alleles.
For there was also an epitope predicted in other parts of the protein. There was an overall loss of 14 predicted epitopes.
No changes in the number One epitope (MHHHHHHTAASDNFQ, binding to DRB1*08:18) of covered alleles. was predicted for the Meth-His tag in Mtb72F. There were also other epitopes predicted for this allele.

Addition of 2-amino acid hinge sequences at the No changes in the number junction sites between Mtb32c and Mtb39A (EF), of covered alleles. and between Mtb39A and Mtb32 $(\mathrm{DI})$.

Adding the EF and DI sequences resulted in 13 and 5 additional predicted epitopes, binding to 43 and 31 alleles, respectively. However, the number of alleles with at least one predicted epitope did not change. 
Table 5 Comparison of experimentally-defined and predicted 15-mer HLA class II-binding peptides for three DRB1 alleles

\begin{tabular}{|c|c|c|c|c|c|c|c|c|c|}
\hline \multirow[t]{2}{*}{ Performance algorithm } & \multicolumn{3}{|l|}{ DRB1*0101 (DR1) } & \multicolumn{3}{|l|}{ DRB1*1501 (DR2) } & \multicolumn{3}{|l|}{ DRB1*0401 (DR4) } \\
\hline & Mtb32A & Mtb39A & Mtb72F & Mtb32A & Mtb39A & Mtb72F & Mtb32A & Mtb39A & Mtb72F \\
\hline & INAFSVGSGQTYGVD & VNEAEYGEMWAQDAA & & LSQDRFADFPALPLD & MLKGFAPAAAAQAVQ & & $\underline{\text { LNGLIQFDAAIQPGD }}$ & TAYGLTVPPPVIAEN & \\
\hline & GSGQTYGVDWGYDR & $\underline{\text { AYETAYGLTVPPPVI }}$ & & DRFADFPALPLDPSA & KTVSPHRSPISNMVS & & NFQLSQGGQGFAIPI & WWGLTVGSWIGSSA & \\
\hline & ATDINAFSVGSGQTY & AEYGEMWAQDAAAMF & & APAQAAPPALSQDRF ${ }^{\mathbf{a}}$ & MSSLGSSLGSSGLGG & & LNGHHPGDVISVTWQ & GLTVGSWIGSSAGLM & \\
\hline & $\underline{\text { YDRTQDVAVLQLRGA }}$ & GEMWAQDAAAMFGYA & & & SSAGLMVAAASPYVA & & LTNNHVIAGATDINA & GVAANLGRAASVGSL & \\
\hline & $\underline{\text { VAVLQLRGAGGLPSA }}$ & $\underline{\text { AAAAYETAYGLTVPP }}$ & & & & & QTYGVDWGYDRTQD & ANLGRAASVGSLSVP & \\
\hline & GGQGGTPRAVPGRW & $\underline{\text { ASVGSLSVPQAWAAA }}$ & & & & & VPGRWVALGQTVQAS & AAAAYETAYGLTVPP & \\
\hline & QTYGVDWGYDRTQD & $\underline{\text { VVWGLTVGSWIGSSA }}$ & & & & & IPIGQAMAIAGQIRS & ASAFQSWWGLTVGS & \\
\hline & FSVGSGQTYGVDWG & VRVAAAAYETAYGLT & & & & & NGARVQRWGSAPAA & VTPAARALPLTSLTS & \\
\hline & IPIGQAMAIAGQIRS & AIAVNEAEYGEMWAQ & & & & & INAFSVGSGQTYGVD & $\underline{\text { LMILIATNLLGQNTP }}$ & \\
\hline & & $\underline{\text { MLKGFAPAAAAQAVQ }}$ & & & & & RVQRVVGSAPAASLG & VAAASPYVAWMSVTA & \\
\hline & & AENRAELMILIATNL & & & & & AGQIRSGGGSPTVHI & ASPYVAWMSVTAGQA & \\
\hline & & ASAFQSVVWGLTVGS & & & & & GSGQTYGVDWGYDR & VGSWIGSSAGLMVAA & \\
\hline & & FSAASAFQSVVWGLT & & & & & $\underline{\text { LQLRGAGGLPSAAIG }}$ & AENRAELMILIATNL & \\
\hline & & SSAGLMVAAASPYVA & & & & & IAGATDINAFSVGSG & SASLVAAAQMWDSVA & \\
\hline & & & & & & & RWALGQTVQASDSL & SSAGLMVAAASPYVA & \\
\hline & & & & & & & YDRTQDVAVLQLRGA & LPPEINSARMYAGPG & \\
\hline & & & & & & & FSVGSGQTYGVDWG & GQAELTAAQVRVAAA & \\
\hline & & & & & & & RWGSAPAASLGIST & MLKGFAPAAAAQAVQ & \\
\hline & & & & & & & $\underline{\text { VAVLQLRGAGGLPSA }}$ & AAQVRVAAAAYETAY & \\
\hline & & & & & & & GFAIPIGQAMAIAGQ & YVAWMSVTAGQAELT & \\
\hline & & & & & & & GVDWGYDRTQDVAV & DAAAMFGYAAATATA & \\
\hline & & & & & & & ATDINAFSVGSGQTY & PSSKLGGLWKTVSPH & \\
\hline & & & & & & & PLDPSAMVAQVGPQV & VTAGQAELTAAQVRV & \\
\hline & & & & & & & TQDVAVLQLRGAGGL & AVQTAAQNGVRAMSS & \\
\hline & & & & & & & GGTPRAVPGRWALG & LIATNLLGQNTPAIA & \\
\hline & & & & & & & NHVIAGATDINAFSV & GEMWAQDAAAMFGYA & \\
\hline & & & & & & & GGGSPTVHIGPTAFL & MYAGPGSASLVAAAQ & \\
\hline & & & & & & & GTGIVIDPNGWLTN & VRVAAAAYETAYGLT & \\
\hline & & & & & & & RWSWLLSVLAAVGLG ${ }^{\mathbf{a}}$ & FSAASAFQSVVWGLT & \\
\hline & & & & & & & & AIAVNEAEYGEMWAQ & \\
\hline & & & & & & & & $\underline{\text { AYETAYGLTVPPPVI }}$ & \\
\hline & & & & & & & & ASVGSLSVPQAWAAA & \\
\hline
\end{tabular}


Table 5 Comparison of experimentally-defined and predicted 15-mer HLA class II-binding peptides for three DRB1 alleles (Continued)

\begin{tabular}{|c|c|c|c|c|c|c|c|c|c|}
\hline $\begin{array}{l}\text { Total binders determined } \\
\text { in vitro }\end{array}$ & 9 & 14 & 23 & 3 & 4 & 6 & 29 & 32 & 60 \\
\hline Total non-binders ${ }^{\mathrm{b}}$ & 106 & 113 & 202 & 112 & 123 & 219 & 86 & 95 & 165 \\
\hline $\begin{array}{l}\text { True predicted binders } \\
\text { (TP) }\end{array}$ & 5 & 12 & 17 & 0 & 2 & 2 & 4 & 13 & 17 \\
\hline $\begin{array}{l}\text { True predicted } \\
\text { non-binders (TN) }\end{array}$ & 45 & 28 & 68 & 102 & 108 & 197 & 77 & 70 & 134 \\
\hline $\begin{array}{l}\text { Sensitivity prediction } \\
\text { algorithm }\end{array}$ & $55 \%$ & $86 \%$ & $74 \%$ & $0 \%$ & $50 \%$ & $33 \%$ & $14 \%$ & $41 \%$ & $28 \%$ \\
\hline $\begin{array}{l}\text { Specificity prediction } \\
\text { algorithm }\end{array}$ & $42 \%$ & $25 \%$ & $34 \%$ & $91 \%$ & $88 \%$ & $90 \%$ & $90 \%$ & $74 \%$ & $81 \%$ \\
\hline
\end{tabular}

Listed epitopes were derived from ref. [18] and experimentally found to bind MCH Class II molecules. Peptides predicted by NetMHCpan-3.0 in the current study are underlined and highlighted in bold. TP True positives. In ref. Listed epitopes were derived from ref. [18] and experimentally found to bind $\mathrm{MCH}$ Class $1 /$ molecules. Peptides predicted by NetMHCpan-3.0 in the current study are underlined and highlighted in bold. $7 P$ True positives. In
$[18]$ and the current study, data were generated using 15 mer peptides overlapping by 12 mer. TN True negatives. ${ }^{a}$ Sequence does not appear in the Mtb72F recombinant protein. ${ }^{b}$ Length of the Mtb72F sequence is 729 amino acids, containing 225 overlapping15-mer peptides. Mtb32A and Mtb39A contain 115 and 127 overlapping 15-mer peptides 
sequence (the 2-aa insertion at the 162nd base and the 1aa deletions at the 274th base, in repeat regions in 1 and 6 strains, respectively) may be identical to those reported for Arkansas-derived samples in the above study [28]. Of the frameshifts leading to major amino acid changes, as we observed in 3 strains, the one in the avirulent H37Ra strain was consistent with earlier findings [41] and of little clinical relevance, while the one in EAS054 has not been reported previously to the best of our knowledge. Possibly, the number and origin of the strains evaluated here reflect the worldwide prevalence of Mtb more accurately than those used in the referenced studies, which included 225 US and Turkey-derived clinical strains [28], or 16 clinical isolate sequences from a public database [29]. As we have not assessed whether the minor insertions and/or deletions coincide with locations of predicted putative epitopes, the impact of the associated residue changes on the recognition of clinical Mtb strains by T-cell responses induced by Mtb72F or M72 candidate vaccines, is not known.

MHC-II binding peptides in Mtb72F were predicted to cover all DRB1, DRB3/4/5, DQ and DP alleles assessed, except for 2 DRB1 alleles (03:02 and 16:04), 1 DQ and 1 DP allele. Based on the used database, this DP allele (DPA1*02:02-DPB1*05:01) does not occur in the populations assessed, while the DQ allele (DQA1*02:01DQB1*02:01) only prevails in these populations in haplotype with DRB1*07:01, which was predicted to be well covered with binding peptides. Several aspects are important to consider with respect to the implications of the absence of predicted binding peptides for the two DRB1 alleles for the candidate vaccines' potential efficacy in the populations concerned. First, except for the higher frequency of DRB1*03:02 in certain SubSaharan African populations $(\leq 17.2 \%)$ as compared with the worldwide genotypic frequency of $1.1 \%$ [37], the associated allele frequencies were generally low in these populations $(\leq 1.5 \%)$. Moreover, a potential absence of binding peptides for the two DRB1 alleles in these populations may be compensated by peptides present on other HLA-DR, -DP or -DQ alleles, and vice versa. For instance, for the South-African Limpopo Venda population, DRB1*13:02 and DQB1*03:01-03:04 have been associated with TB disease [42]. In this population, the frequency of DRB1*03:02 is relatively high ( $9 \%$ ). The absence of binding peptides for DRB1*03:02 may in this case be compensated by several other alleles prevailing at high frequencies in this population (e.g., DRB1*11, DRB1*03:01, DRB1*13:01, DRB*01 and DQB1*06 and DQB1*05; data retrieved from [36]), for which we predicted high numbers of binding peptides per allele in Mtb72F. It may also be reassuring that in a recent clinical trial, the candidate vaccine M72/ AS01 was able to induce robust $\mathrm{CD} 4^{+} \mathrm{T}$-cell responses in healthy adults in South Africa [22]. Furthermore, it is noted that for other antigens (meningococcal serogroup B proteins) it was shown that a set of only 2 predicted T-cell epitopes could theoretically cover large proportions of over 11 populations worldwide [43]. A confounding factor is that the allele frequencies sourced from a public database may have only partial or local coverage (e.g. cities or ethnic groups), since they are usually based on relatively small sample sizes rather than population statistics. Similarly, since certain alleles specific to susceptible populations have likely been the prime focus of several studies, the database may contain disproportionally high numbers of such alleles.

Although our data showed that the changes introduced in Mtb32A in order to construct Mtb72F may result in an overall reduction of Mtb binding peptides, only two alleles present in the populations assessed (DRB1*03:02 and 16:04) were predicted to have no binding peptide in Mtb72F. The other modifications (addition of hinge sequences and a poly-His tag) were predicted to jointly generate 19 binding peptides, of which one peptide was predicted to be absent in M72. Since non-Mtb epitopes do not contribute to the vaccine's ability to induce Mtb-specific responses, their relatively low frequency in Mtb72F and M72 is reassuring.

Previously, in silico MHC-II binding predictions for 9-mer peptides in Mtb72F have been generated for 34 DRB1 alleles [26, 27]. Comparison of the previous data with the present predictions for 15 -mer peptides and DRB1 alleles revealed up to 37 -fold differences in the allele-matched binding peptide numbers, which can likely for a great part be explained by the different binding peptide lengths assumed by the prediction algorithm. HLA-I and HLA-II binding peptides are composed of 8-10 and 13-17 amino acids respectively [44]. The peptide-binding groove of MCH-II molecules is open-ended, which complicates identification of the 9mer core peptides and the flanking residues (which may also contain predictive information [45]). MHC-II prediction algorithms assuming 9-mer binding peptides do not use information present outside the core, leading to lower accuracy [46]. Consistently, it was shown that including flanking residues among inputs improved the performance of MHC-II prediction methods $[47,48]$. However, even predictions generated by algorithms assuming 15-mer binding peptides may have limited reproducibility, as suggested by the present results. Indeed, comparison of our results for Mtb32A and Mtb39A with allele-matched experimental binding data [18] revealed that the algorithm's sensitivity and specificity were variable and occasionally rather low for Mtb72F (e.g., $28 \%$ ). It is noted though that due to the lack of additional suitable in vitro data, this comparison covered only 3 of the 158 HLA-DRB1 alleles, and none of the DRB3/4/5, DQ and DP alleles assessed in our study. While the observed variability in the 
performance of the algorithm may be a consequence of the limited extent of this evaluation, it could therefore also be inherent to the limitations of the used prediction algorithms for peptide binding in general. Similarly, most algorithms predicting T-cell activation for class II binding peptides display low sensitivity, as less than half of the defined T-cell activating peptides were predicted to be binders [46]. Thus, epitope identification requires an integrated approach, in which in silico binding predictions are complemented with confirmatory biochemical verification of MHC processing and binding, and of the ability of the predicted peptides to induce T-cell responses [49]. Other useful tools are in silico methods that enable linking binding affinity patterns to topological characteristics [50].

Due to the capped MHC-I peptide binding groove, this binding is more specific than binding to MHC-II, and HLA-I binding prediction algorithms are considered relatively accurate $[46,49]$. Indeed, there was only at most a five-fold difference between the previously reported numbers of HLA A- and B-binding peptides in Mtb72F [27] and the numbers per allele computed here.

In the present study, the majority of predicted peptides were presented by $A^{*} 02: 01$, listed in the IEDB among the most frequent HLA class I alleles in regions with high TB incidence [4]. The predicted high affinity-binders were mainly presented by $\mathrm{A}^{* 02: 01}$ and $\mathrm{B} * 07: 02$ rather than A*03:01, consistent with data obtained for a panel of 432 H37Rv-derived peptides [5], while other Mtb proteins (e.g. Mtb8.4, CFP10 and Ag85B) were shown, experimentally or in silico, to be primarily presented by various HLA-B molecules $[51,52]$. Furthermore, our data showing the predicted binding of the Mtb72F peptides APINSATAM, APAAAAQAV, LLGQNTPAI and SSKLGGLWK to B*07:02, B*07:02, $A^{*} 02: 01$ and $A^{*} 03: 01$ respectively corroborates earlier predictions for these peptides [5]. Of note, several previously predicted HLA I-restricted Mtb peptides were experimentally shown to induce $\mathrm{CD} 4^{+}$rather than $\mathrm{CD} 8^{+} \mathrm{T}$ cell responses [53]. Similarly, the HLA-B44-restricted epitope MWAQDAAAMF [16], was also found in two 15-mer Mtb39A peptides that were experimentally defined [18] and predicted here to bind to DRB1*04:01 and/or 01:01.

Last, for cytomegalovirus antigens, it has been suggested that less than 20 uniquely defined HLA-restricted $\mathrm{CD}^{+} \mathrm{T}$ cell epitopes could provide $90 \%$ coverage of the three major ethnic groups [54]. Possibly, the putative class I Mtb epitopes predicted for Mtb72F could also have a wide coverage of populations worldwide, and, given that only minor changes were introduced into Mtb72F to generate M72, this would also apply to M72.

\section{Conclusions}

Mtb72F and M72 sequences were shown to be highly conserved among Mycobacterium strains including MDR and XDR strains, and are thus expected to provide broad coverage of the pathogen population. For Mtb72F, the predicted MHC-II binding peptides covered a wide array of HLA-DRB1, -DQ and -DP alleles, whereby the absence of predicted binding peptides for 2 DRB1 alleles prevailing in some populations with high TB burden may be compensated by peptides binding to other HLA loci. MHC-I binding peptides were predicted for each HLA-A/B allele assessed. Yet, discrepancies between the current predictions and experimental HLA-binding data underscore the limitations inherent in interpreting in silico predictions for HLA class II-restricted peptides. Experimental epitope verification, by determination of the HLA-binding capacity of synthetic peptides and of their correct processing, remains valuable. Also, their immunogenicity could be confirmed by identification of $\mathrm{T}$ cells specific for the epitope, using PBMCs from HLAtyped subjects.

\section{Methods}

Mtb72F and $\mathrm{M72}$ vaccine antigens

Mtb72F is a 729-aa, 72-kDa polyprotein derived from Mtb32A and Mtb39A (encoded by Rv0125 and Rv1196, respectively). The full nucleotide and deduced amino acid sequences of Mtb32A, Mtb39A and Mtb72F have been described [12-14]. Mtb72F has been constructed in the linear order (starting at the amino end): carboxyl-terminal portion of Mtb32A (Mtb32 ${ }_{C}$ ) - full-length ORF of Mtb39A - N-terminal fragment of Mtb32A excluding the signal sequence $\left(\mathrm{Mtb} 32_{\mathrm{N}}\right)$. Since the Mtb32A sequence has been split upstream and downstream of the TAAS sequence linking the $M \operatorname{tb} 32_{\mathrm{C}}$ and $\mathrm{Mtb} 32_{\mathrm{N}}$ sequences, TAAS is present in Mtb72F at the $\mathrm{N}$-terminal end of $\mathrm{Mtb} 32_{\mathrm{C}}$ as well as at the C-terminal end of Mtb32 $2_{\mathrm{N}}$. In summary, the following changes were made compared to the native Mtb32A protein: (i) addition of nucleotide sequences encoding a poly-His tag (MHHHHHH) at the Mtb72F N-terminus; (ii) deletion of the 32-aa signal sequence (MSNSRRRSLRWSWLLSVLAAVGLGLATAPA QA) located at the Mtb32 N-terminus; (iii) addition of two hinge sequences (an EcoRI restriction site, located between Mtb32 ${ }_{\mathrm{C}}$ and Mtb39A, and an EcoRV restriction site between Mtb39A and Mtb32 ${ }_{\mathrm{N}}$ ) resulting in the $\mathrm{EF}$ and DI residues in Mtb72F, respectively (one at each junction site).

M72 was generated by introducing a point mutation in Mtb72F, thereby replacing Ser $_{706}$ by Ala ${ }_{706}$, and by changing the poly-His tag of Mtb72F into the MHH sequence present in M72.

\section{Sequence alignments and similarity analyses}

Using the BLAST program [55], Mtb protein sequences available in the NCBI GenPept database (accessed February 
2013) were screened for the presence of Mtb32A and Mtb39A sequence. The selection of 44 and 42 genomes for Mtb32A and Mtb39A respectively comprised the laboratory strain H37Ra, as well as $M$. africanum and clinical isolates from presently available Mtb strains including MDR, XDR and pre-XDR strains (i.e., Mtb Beijing family strains and North and South-American, African, Asian, and European strains). To the extent the information was available, the strains were matched with a detailed molecular phylogeny of the Mtb complex [30], which divides the global population structure of Mtb strains in 6 main phylogeographical lineages. Our selection included predominant strains from each main lineage, with the exception of Lineage 3 (India/ East Africa) for which no strains were included (Table 1).

Complete genomes, or assembled partial genomes when validated and/or complete genomes were unavailable, were downloaded from the GenBank database. For the 40 strains for which both the Mtb32A and Mtb39A sequences were available, the genomes were used for the construction of Mtb72F and M72 as described above (see 'Mtb72F and M72 vaccine antigens'), excluding the additional residues at the junction sites and the Mtb72F/ M72 poly-His tag). To ensure that the used sequence was specific for $m t b 39$, rather than for one of the known mtb39 paralogs (ppe19 and ppe60), a fragment containing Mtb39A with PE13 upstream, and ESAT-6-like (esxL) downstream, was extracted from the TB genomes. Mtb39A nucleotide sequences from 42 strains were translated into amino acid sequences, which were subsequently compared by multiple alignments using ClustalX software. For Mtb72F and M72, all protein sequences had very similar or identical lengths. Similarity analyses for Mtb32A, Mtb39A, Mtb72F and M72 were done by multiple alignment comparisons. Percentage identity between the sequences was defined as 'number of identical residues / length of alignment) x 100'. The data for Mtb39A were also analysed separately for the presence of nucleotide and amino acid changes (insertions, deletions and/or frameshifts).

\section{$\mathrm{DRB}, \mathrm{DP}$ and $\mathrm{DQ}$ allele frequencies}

MHC-II peptide binding predictions were done for HLADRB1, DRB3/4/5, DQ and DP alleles, whereby the DRB1 allele frequencies characterizing the populations in each region were extracted from the 'Allele"frequencies in Worldwide Populations' database [36] (http://www.allelefrequencies.net; accessed November 2013). The 158 DRB1 alleles evaluated (including the common alleles 01:01, 03:01, 04:01, 07:01, 11:01 and 15:01) comprised 146, 47, 25, 42 and 66 alleles present in populations in China, North India, Southern India, North-Eastern India and Sub-Saharan Africa (native population), respectively. India and China, and 9 Sub-Saharan African countries are among the 22 high-TB burden regions defined by the World Health Organization [56]. The discrimination of
Northern, Southern, and North-Eastern Indian regions was based on the local prevalent population structures, while for the Chinese and Sub-Saharan African regions insufficient information was available to apply further detailing. The 8 HLA-DQ molecules assessed contained the 6 molecules that reportedly are present in over $85 \%$ of populations worldwide [32], and the 6 HLA-DP molecules assessed contained the 5 HLA-DP molecules that reportedly are present in $>90 \%$ of populations worldwide [33] (see Table 3). Frequencies of alleles for which no binding peptides were predicted in this study were extracted from the above-mentioned public database [36]; accessed 26 January 2015). HLA-I binding predictions were done for $A^{*} 02: 01, A * 03: 01$ and $B * 07: 02$, which were selected by the developers of the NetMHCpan-2.2 algorithm to represent the supertypes A02, A03 and B07 respectively [38, 57]. Collectively these supertypes have been reported to cover $83-88 \%$ of 5 major ethnic groups worldwide [39].

\section{HLA class II-binding peptide predictions for DRB, DP and DQ alleles}

HLA-II binding predictions were generated for 15-mer peptides (overlapping by 14 amino acids) of Mtb32A, Mtb39A and Mtb72F using NetMHCIIpan-3.0 [58], and for the 158 DRB1, 6 DRB3/4/5, 6 DP and 8 DQ alleles described. The Mtb72F construct was produced in silico as described above (see ' $M t b 72 F$ and M72 vaccine antigens') with exclusion of the poly-His sequence. In addition, the potential impact of the alterations introduced for the Mtb72F and M72 constructions on the prediction results was evaluated. For DRB1*01:01, DRB1*04:01 and DRB1*15:01, data were also generated using 15 mer peptides overlapping by 12 amino acids. Results were compared with previously published experimental data for 15mer Mtb peptides (overlapping by 12 amino acids) binding to these 3 alleles [18], using the Mtb72F sequence with exclusion of the hinge sequences and poly-His tag. As recommended by the program, a peptide was identified as a 'strong binder' if the binding affinity $\left[\mathrm{IC}_{50}\right]$ was below 50 $\mathrm{nM}$ or as a 'weak binder' if the $\mathrm{IC}_{50}$ was $>50 \mathrm{nM}$ but $<500$ $\mathrm{nM}$. The total numbers of epitopes shown in this study represent the sum of the strong and the weak binders.

\section{HLA class I-binding peptide predictions}

MHC-I-binding predictions for the A*02:01, A*03:01 and $\mathrm{B}^{*}$ 07:02 alleles were done for 9-mer peptides in Mtb72F using NetCTLpan-1 [59], NetMHCpan-2.2 [57], SVMHC, Syfpeithi [2], EpiJen [60], nHLA-pred (nHLAcompred and nHLA-aanpred) and NetCTL-1.2 [61]. Results are presented as the individual outputs of NetCTLpan-1 and NetMHCpan-2.2 (which have been reported as having a higher accuracy [57, 59]) or as grouped outputs for the other programs used. Positive results generated by NetCTLpan were assigned ' $*$ ' if the 
putative epitopes were correctly processed (proteasome and transporters associated with antigen processing [TAP]) and bound to the MHC. For all other programs, positive results were classified based on binding strength to the $\mathrm{MHC}$, either as strong or weak binders ('SB' or 'WB', respectively) based on the author-recommended cut-offs.

\section{Availability of supporting data}

The data sets supporting the results of this article are included in Tables 1, 2, 3, 4 and 5 and Fig. 1 within the article, and in its Additional files 1, 2 and 3. The 15-mer class II-binding peptides listed in Table 5 were originally derived from Supplementary Table S1 in ref. [18], and allocated to Mtb32A or Mtb39A by the authors of the latter publication.

\section{Ethics statement}

The authors declare that this study does not involve any ethical issues, since no animal or human experiments were performed as part of the research for this manuscript.

\section{Additional files}

\section{Additional file 1: Predicted HLA class II binding peptides in \\ Mtb72F. (XLSX $15 \mathrm{~kb}$ )}

Additional file 2: Frequency of alleles without predicted binding peptides in the populations assessed. (PDF $66 \mathrm{~kb}$ )

Additional file 3: Predicted 9-mer HLA class I peptide binding regions in Mtb72F. (XLSX $13 \mathrm{~kb}$ )

\section{Competing interests}

The authors have declared the following conflicts of interest: all authors are employees of the GSK group of companies, and EJ, PM and JLR report ownership of GSK shares.

\section{Authors' contributions}

All authors participated in the design, analysis, and interpretation of the study results. MCM and JLR participated in the implementation of the study. All authors were involved in drafting the manuscript or revising it critically for important intellectual content. All authors had full access to the data and approved the manuscript before it was submitted by the corresponding author.

\section{Authors' information}

Not applicable.

\section{Acknowledgements}

The authors thank Markus Maeurer (Microbiology and Tumor Biology Center, Karolinska Institute, Stockholm, Sweden) for kindly providing the allocations to Mtb32A or Mtb39A of the 15-mer class II-binding peptides published in [18]. They also thank Marie-Ange Demoitié, Scott Goodall, Guy Baudoux and Stéphane Temmerman for their critical review of the manuscript and/or helpful discussions, Ellen Oe (XPePharma on behalf of GSK) for providing scientific writing services in the manuscript's development, and Ulrike Krause (GSK Vaccines, Belgium) for publication management.

\section{Funding}

GlaxoSmithKline Biologicals SA was the funding source and was involved in all stages of the study conduct and analysis. The costs associated with the development and publishing of the manuscript, including scientific writing assistance, were also covered by GlaxoSmithKline Biologicals SA.
Received: 9 March 2015 Accepted: 8 September 2015

Published online: 22 October 2015

\section{References}

1. Kaufmann SH, Lange C, Rao M, Balaji KN, Lotze M, Schito M, et al. Progress in tuberculosis vaccine development and host-directed therapies-a state of the art review. Lancet Respir Med. 2014;2:301-20.

2. Rammensee H, Bachmann J, Emmerich NP, Bachor OA, Stevanović S. SYFPEITHI: database for MHC ligands and peptide motifs. Immunogenetics. 1999;50:213-9.

3. Vita R, Zarebski L, Greenbaum JA, Emami H, Hoof I, Salimi N, et al. The Immune Epitope Database 2.0. Nucleic Acids Res. 2010;38:D854-62.

4. Blythe MJ, Zhang Q, Vaughan K, de Castro Jr R, Salimi N, Bui HH, et al. An analysis of the epitope knowledge related to Mycobacteria. Immunome Res. 2007:3:10.

5. Tang ST, van Meijgaarden KE, Caccamo N, Guggino G, Klein MR, van Weeren $P$, et al. Genome-based in silico identification of new Mycobacterium tuberculosis antigens activating polyfunctional $\mathrm{CD} 8^{+} \mathrm{T}$ cells in human tuberculosis. J Immunol. 2011;186:1068-80.

6. Axelsson-Robertson R, Magalhaes I, Parida SK, Zumla A, Maeurer M. The immunological footprint of Mycobacterium tuberculosis T-cell epitope recognition. J Infect Dis. 2012;205 Suppl 2:S301-15.

7. Sampson SL. Mycobacterial PE/PPE proteins at the host-pathogen interface. Clin Dev Immunol. 2011;2011:497203.

8. Mukhopadhyay S, Balaji KN. The PE and PPE proteins of Mycobacterium tuberculosis. Tuberculosis (Edinb). 2011;91:441-7.

9. Bhat KH, Ahmed A, Kumar S, Sharma P, Mukhopadhyay S. Role of PPE18 protein in intracellular survival and pathogenicity of Mycobacterium tuberculosis in mice. PLOS ONE. 2012;7:e52601.

10. Nair S, Pandey AD, Mukhopadhyay S. The PPE18 protein of Mycobacterium tuberculosis inhibits NF-kB/rel-mediated proinflammatory cytokine production by upregulating and phosphorylating suppressor of cytokine signaling 3 protein. J Immunol. 2011;186:5413-24.

11. Sayes F, Sun L, Di Luca M, Simeone R, Degaiffier N, Fiette L, et al. Strong immunogenicity and cross-reactivity of Mycobacterium tuberculosis ESX-5 type VII secretion: encoded PE-PPE proteins predicts vaccine potential. Cell Host Microbe. 2012;11:352-63.

12. Skeiky YA, Alderson MR, Ovendale PJ, Guderian JA, Brandt L, Dillon DC, et al. Differential immune responses and protective efficacy induced by components of a tuberculosis polyprotein vaccine, Mtb72F, delivered as naked DNA or recombinant protein. J Immunol. 2004;172:7618-28.

13. Skeiky YAW, Lodes MJ, Guderian JA, Mohamath R, Bement T, Alderson MR, et al. Cloning, expression, and immunological evaluation of two putative secreted serine protease antigens of Mycobacterium tuberculosis. Infect Immun. 1999;67:3998-4007.

14. Dillon DC, Alderson MR, Day CH, Lewinsohn DM, Coler R, Bement T, et al. Molecular characterization and human T-cell responses to a member of a novel Mycobacterium tuberculosis mtb39 gene family. Infect Immun. 1999;67:2941-50.

15. Spertini F, Audran R, Lurati F, Ofori-Anyinam O, Zysset F, Vandepapelière $P$, et al. The candidate tuberculosis vaccine Mtb72F/AS02 in PPD positive adults: A randomized controlled phase I/II study. Tuberculosis (Edinb). 2013;93:179-88.

16. Lewinsohn DA, Lines RA, Lewinsohn DM. Human dendritic cells presenting adenovirally expressed antigen elicit Mycobacterium tuberculosis-specific CD8 ${ }^{+}$T cells. Am J Respir Crit Care Med. 2002;166:843-8.

17. Irwin SM, Izzo AA, Dow SW, Skeiky YA, Reed SG, Alderson MR, et al. Tracking antigen-specific CD8 T lymphocytes in the lungs of mice vaccinated with the Mtb72F polyprotein. Infect Immun. 2005;73:5809-16.

18. Gaseitsiwe S, Valentini D, Mahdavifar S, Reilly M, Ehrnst A, Maeurer M. Peptide microarray-based identification of Mycobacterium tuberculosis epitope binding to HLA-DRB1*0101, DRB1*1501, and DRB1*0401. Clin Vaccine Immunol. 2010;17:168-75

19. Leroux-Roels I, Forgus S, De Boever F, Clement F, Demoitié M-A, Mettens P, et al. Improved $\mathrm{CD}^{+} \mathrm{T}$ cell responses to Mycobacterium tuberculosis in PPD-negative adults by M72/AS01 as compared to the M72/AS02 and Mtb72F/ASO2 tuberculosis candidate vaccine formulations: A randomized trial. Vaccine. 2013:31:2196-206.

20. Von Eschen K, Morrison R, Braun M, Ofori-Anyinam O, De Kock E, Pavithran $P$, et al. The candidate tuberculosis vaccine Mtb72F/AS02A: Tolerability and immunogenicity in humans. Hum Vaccin. 2009;5:475-82.

21. Leroux-Roels I, Leroux-Roels G, Ofori-Anyinam O, Moris P, De Kock E, Clement $F$, et al. Evaluation of the safety and immunogenicity of two antigen 
concentrations of the Mtb72F/ASO2 $\mathrm{A}$ candidate tuberculosis vaccine in Purified Protein Derivative-negative adults. Clin Vaccine Immunol. 2010;17:1763-71.

22. Day $\mathrm{CL}$, Tameris M, Mansoor N, van Rooyen M, de Kock M, Geldenhuys $\mathrm{H}$, et al. Induction and regulation of T-cell immunity by the novel TB vaccine M72/AS01 in South African adults. Am J Respir Crit Care Med. 2013:188:492-502

23. Montoya J, Solon JA, Cunanan SR, Acosta L, Bollaerts A, Moris P, et al. A randomized, controlled dose-finding Phase II study of the M72/AS01 candidate tuberculosis vaccine in healthy PPD-positive adults. J Clin Immunol. 2013;33:1360-75.

24. Thacher EG, Cavassini M, Audran R, Thierry A-C, Bollaerts A, Cohen J, et al. Safety and immunogenicity of the M72/AS01 candidate tuberculosis vaccine in HIV-infected adults on combination antiretroviral therapy: a phase I/II, randomized trial. AIDS. 2014;28:1769-81.

25. Idoko OT, Owolabi OA, Owiafe PK, Moris P, Odutola A, Bollaerts A, et al. Safety and immunogenicity of the M72/ASO1 candidate tuberculosis vaccine when given as a booster to BCG in Gambian infants: An open-label randomized controlled trial. Tuberculosis (Edinb). 2014;94:564-78.

26. McNamara LA, He Y, Yang Z. Using epitope predictions to evaluate efficacy and population coverage of the Mtb72f vaccine for tuberculosis. BMC Immunol. 2010;11:18

27. Davila J, McNamara LA, Yang Z. Comparison of the predicted population coverage of tuberculosis vaccine candidates Ag85B-ESAT-6, Ag85B-TB10.4, and Mtb72f via a bioinformatics approach. PLOS ONE. 2012;7:e40882.

28. Hebert AM, Talarico S, Yang D, Durmaz R, Marrs CF, Zhang L, et al. DNA polymorphisms in the pepA and PPE18 genes among clinical strains of Mycobacterium tuberculosis: implications for vaccine efficacy. Infect Immun. 2007;75:5798-805

29. McEvoy CR, Cloete R, Müller B, Schürch AC, van Helden PD, Gagneux S, et al. Comparative analysis of Mycobacterium tuberculosis pe and ppe genes reveals high sequence variation and an apparent absence of selective constraints. PLoS ONE. 2012;7:e30593.

30. Smith NH, Hewinson RG, Kremer K, Brosch R, Gordon SV. Myths and misconceptions: the origin and evolution of Mycobacterium tuberculosis. Nat Rev Microbiol. 2009;7:537-44.

31. McKinney DM, Southwood S, Hinz D, Oseroff C, Lindestam Arlehamn CS, Schulten $\mathrm{V}$, et al. A strategy to determine HLA class II restriction broadly covering the DR, DP, and DQ allelic variants most commonly expressed in the general population. Immunogenetics. 2013;65:357-70.

32. Sidney J, Steen A, Moore C, Ngo S, Chung J, Peters B, et al. Divergent motifs but overlapping binding repertoires of six HLA-DQ molecules frequently expressed in the worldwide human population. J Immunol. 2010;185:4189-98.

33. Sidney J, Steen A, Moore C, Ngo S, Chung J, Peters B, et al. Five HLA-DP molecules frequently expressed in the worldwide human population share a common HLA supertypic binding specificity. J Immunol. 2010;184:2492-503.

34. Selvaraj P, Uma H, Reetha AM, Kurian SM, Xavier T, Prabhakar R, et al. HLA antigen profile in pulmonary tuberculosis patients and their spouses. Indian J Med Res. 1998;107:155-8.

35. Ravikumar M, Dheenadhayalan V, Rajaram K, Lakshmi SS, Kumaran PP, Paramasivan CN, et al. Associations of HLA-DRB1, DQB1 and DPB1 alleles with pulmonary tuberculosis in south India. Tuber Lung Dis. 1999;79:309-17.

36. Gonzalez-Galarza FF, Christmas S, Middleton D, Jones AR. Allele frequency net: a database and online repository for immune gene frequencies in worldwide populations. Nucleic Acids Res. 2011;39:D913-9.

37. Lindestam Arlehamn CS, Sette A. Definition of CD4 immunosignatures associated with MTB. Front Immunol. 2014;5:124.

38. Lund $O$, Nielsen M, Kesmir C, Petersen AG, Lundegaard C, Worning P, et al. Definition of supertypes for HLA molecules using clustering of specificity matrices. Immunogenetics. 2004;55:797-810.

39. Sette A, Sidney J. Nine major HLA class I supertypes account for the vast preponderance of HLA-A and -B polymorphism. Immunogenetics. 1999;50:201-12.

40. Comas I, Chakravartti J, Small PM, Galagan J, Niemann S, Kremer K, et al. Human T cell epitopes of Mycobacterium tuberculosis are evolutionarily hyperconserved. Nat Genet. 2010;42:498-503.

41. Zheng H, Lu L, Wang B, Pu S, Zhang X, Zhu G, et al. Genetic basis of virulence attenuation revealed by comparative genomic analysis of Mycobacterium tuberculosis strain H37Ra versus H37Rv. PLoS ONE. 2008:3:e2375.

42. Lombard Z, Dalton DL, Venter PA, Williams RC, Bornman L. Association of $\mathrm{HLA}-\mathrm{DR}$, $-\mathrm{DQ}$, and vitamin $\mathrm{D}$ receptor alleles and haplotypes with tuberculosis in the Venda of South Africa. Hum Immunol. 2006;67:643-54.
43. Gupta SK, Smita S, Sarangi AN, Srivastava M, Akhoon BA, Rahman Q, et al. In silico CD4+ T-cell epitope prediction and HLA distribution analysis for the potential proteins of Neisseria meningitidis Serogroup B - a clue for vaccine development. Vaccine. 2010;28:7092-7.

44. Chicz RM, Urban RG, Lane WS, Gorga JC, Stern L, Vignali DA, et al Predominant naturally processed peptides bound to HLA-DR1 are derived from MHC-related molecules and are heterogeneous in size. Nature. 1992;358:764-8.

45. Godkin AJ, Smith KJ, Willis A, Tejada-Simon MV, Zhang J, Elliott T, et al. Naturally processed HLA class II peptides reveal highly conserved immunogenic flanking region sequence preferences that reflect antigen processing rather than peptide-MHC interactions. J Immunol. 2001:166:6720-7.

46. Wang P, Sidney J, Dow C, Mothé B, Sette A, Peters B. A systematic assessment of MHC class II peptide binding predictions and evaluation of a consensus approach. PLoS Comput Biol. 2008;4:e1000048.

47. Chang ST, Ghosh D, Kirschner DE, Linderman JJ. Peptide length-based prediction of peptide-MHC class II binding. Bioinformatics. 2006;22:2761-7.

48. Nielsen M, Lundegaard C, Lund O. Prediction of MHC class II binding affinity using SMM-align, a novel stabilization matrix alignment method. BMC Bioinformatics. 2007:8:238.

49. Lundegaard C, Lund O, Nielsen M. Predictions versus high-throughput experiments in T-cell epitope discovery: competition or synergy? Expert Rev Vaccines. 2012;11:43-54.

50. Bremel RD, Homan EJ. Recognition of higher order patterns in proteins: immunologic kernels. PLoS ONE. 2013;8:e70115.

51. Lewinsohn DA, Winata E, Swarbrick GM, Tanner KE, Cook MS, Null MD, et al. Immunodominant tuberculosis CD8 antigens preferentially restricted by HLA-B. PLOS Pathog. 2007;3:e127.

52. Vani J, Shaila MS, Chandra NR, Nayak R. A combined immuno-informatics and structure-based modeling approach for prediction of $T$ cell epitopes of secretory proteins of Mycobacterium tuberculosis. Microbes Infect. 2006;8:738-46.

53. Wang M, Tang ST, Stryhn A, Justesen S, Larsen MV, Dziegiel MH, et al. Identification of $\mathrm{MHC}$ class II restricted T-cell-mediated reactivity against MHC class I binding Mycobacterium tuberculosis peptides. Immunology. 2011;132:482-91.

54. Longmate J, York J, La Rosa C, Krishnan R, Zhang M, Senitzer D, et al. Population coverage by HLA class-I restricted cytotoxic T-lymphocyte epitopes. Immunogenetics. 2001;52:165-73.

55. Altschul SF, Gish W, Miller W, Myers EW, Lipman DJ. Basic local alignment search tool. J Mol Biol. 1990;215:403-10.

56. World Health Organization. Global tuberculosis report 2014. Geneva: WHO Press; 2014

57. Lundegaard C, Lund O, Nielsen M. Prediction of epitopes using neural network based methods. J Immunol Methods. 2011;374:26-34.

58. Karosiene E, Rasmussen M, Blicher T, Lund O, Buus S, Nielsen M. NetMHCllpan3.0, a common pan-specific MHC class II prediction method including all three human MHC class II isotypes, HLA-DR, HLA-DP and HLA-DQ. Immunogenetics. 2013;65:711-24.

59. Stranzl T, Larsen MV, Lundegaard C, Nielsen M. NetCTLpan: pan-specific MHC class I pathway epitope predictions. Immunogenetics. 2010;62:357-68.

60. Doytchinova IA, Guan P, Flower DR. EpiJen: a server for multistep T cell epitope prediction. BMC Bioinformatics. 2006;7:131

61. Larsen MV, Lundegaard C, Lamberth K, Buus S, Lund O, Nielsen M. Large-scale validation of methods for cytotoxic T-lymphocyte epitope prediction. BMC Bioinformatics. 2007:8:424 